\title{
Classify Epileptic EEG Signals Using Extreme Support Vector Machine for Ictal and Muscle Artifact Detection
}

\author{
Baiq Siska Febriani Astuti, Santi Wulan Purnami, R. Mohamad Atok, Wardah Rahmatul Islamiyah, Diah \\ Puspito Wulandari, and Anda Iviana Juniani
}

\begin{abstract}
EEG signals aids in diagnosing various wave signals recorded by the activities of the brain. It also produces unavoidable artifacts, in the recording process. The purpose of this study therefore is to detect ictal and artefact signals, with the aim of reducing interpretation errors especially those related to the muscle which are quite difficult to distinguish. The data used are EEG signal recording results obtained from Rumah Sakit Universitas Airlangga. It consisted of two classes, namely ictal and muscle artefact. The signal decomposition method used is a wavelet transform, known as DWT. While the extraction feature utilized, consist of quartile, maximum, minimum, mean and standard deviation. This study also utilized the SVM with linear, polynomial, RBF and ELM (ESVM) kernels. Research results shows that the ESVM classification time is faster than the SVM and other kernels. However, the values of accuracy, sensitivity, specificity and AUC are not better.
\end{abstract}

Keywords-ESVM, SVM, wavelet transform, ICTAL, muscle artifact, epilepsy.

\section{INTRODUCTION}

Epilepsy is a complex collection of brain symptoms which involves varied manifestations due to occurrence of various matters. It is defined as a seizure, which is a temporary event caused by excessive or non-neuronal activity in the brain [1]. Epilepsy is detected by utilizing Electroencephalogram (EEG), a complex signal that carries information from the brain and human nerve functions. EEG signals helps in diagnosing seizures, where each pattern differs from another. Before now, its visual assessment recordings were subjectively acquired from professionals trained to detect epilepsy like a doctor. The information is used for clinical diagnosis and is likely to be used to treat patients suffering from epilepsy. EEG produces various signal waves resulting from recording brain activity. The signal produced has different amplitude in ictal, preictal, postictal, aura, and in open/closed eyes. In addition, the EEG produces waves of

Manuscript received July 14, 2019; revised May 3, 2020. This research was supported by Kementerian Riset, Teknologi, dan Pendidikan Tinggi Republik Indonesia (Grant No:924/PKS/ITS/2018). Classify Epileptic EEG Signals using Extreme Support Vector Machine for Ictal and Muscle Artifact Detection.

Baiq Siska Febriani Astuti, Santi Wulan Purnami, R. Mohamad Atok, and Diah Puspito Wulandari are with the Institut Teknologi Sepuluh Nopember, Indonesia (e-mail: baiqsiskafebriani@gmail.com, santi_wp@its.ac.id, moh_atok@statistics.its.ac.id, diah@te.its.ac.id).

Wardah Rahmatul Islamiyah is with the University of Airlangga, Indonesia (e-mail: wri1905@gmail.com).

Anda Iviana Juniani is with the Shipbuilding Institute of Polytechnic, Indonesia (e-mail: anda.iviana@ppns.ac.id). artifacts that are generally caused by patients like eye, muscle, and heart movements using machines that release electrodes. Signal artifacts tend to have large amplitude and are often mistaken for a seizure (ictal).

The appearance of artefact signals during recording using EGG is quite difficult to withdraw. It resembles various types of EEG signal patterns. Therefore, it makes misunderstandings to regularly occur in signal interpretation [2]. Varieties of signal artifacts resemble a seizure signal. Therefore, in this study, the researchers aim at detecting the muscle artifact and ictal signals.

Support Vector Machine (SVM) and Artificial Neural Network (ANN) are often applied to solve the problem of EEG signal classification [3]-[9]. Based on several previous studies, both techniques are used with various types of preprocessing methods to produce a good performance in solving problems related to epilepsy cases. One of such techniques is the Single Hidden Layer Feed Forward Network (SLFNs), which comprises of methods used to minimize training error and the norm of output weight within Extreme Learning Machines (ELM) [10].

The classification process using the SVM method requires quite a long time to analyze, however, it is one good method that produces high accuracy values when conducting classifications. Therefore, this study, aims to improve the performance of the SVM method to be more effective and efficient, by combining it with the famous ELM technique with high processing speed.

\section{LITERATURE REVIEW}

\section{A. Wavelet Transform}

A wave is ordinarily described as an oscillating function of time or space such as sinusoid. A wavelet is a "small wave" which has its energy contemplated in time to give a device for analysis of transient, nonstationary, or time-varying appearances [11]. Some of the mother wavelets are commonly utilized such as db wavelet, morlet wavelet, haar wavelet, mexican hat wavelet, coifiet wavelet etc. The wavelet transforms protrude in terms of algorithmic elegance and efficiency [12]. Wavelet transform is a tool used to cut data or functions into different frequency components and study each component with a resolution harmonized to its scale [13]. Wavelet transform formula as follows [14]:

$$
\psi_{a, b}(t)=|a|^{-\frac{1}{2}} \psi\left(\frac{t-b}{a}\right)
$$


where $a$ is the scaling parameter and $\mathrm{b}$ is the shifting parameter that determines the location of the time from the wavelet. The function $\psi_{(a, b)}$ is defined as a temporary wavelet $\psi$ also called the mother wavelet [13].

Wavelet transforms are grouped into two, namely Continuous Wavelet Transform (CWT) and Discrete Wavelet Transform (DWT). The CWT coefficients are evaluated for a continuous variation (infinitesimal additions) both of factors namely translation and dilation. According of equation (1), the CWT can be defined as follows [4-5]:

$$
C W T(a, b)=\int_{-\infty}^{\infty} x(t) \frac{1}{\sqrt{|a|}} \psi\left(\frac{t-b}{a}\right) d t
$$

where $\psi, a, b$, and $x(t)$ are the wavelet function scaling, shifting parameters and signal to be processed, respectively. Assuming the function scaling and shifting parameters are converted to the power of two, the wavelet analysis will be more valuable. Therefore, DWT is defined below as follows [4-5]:

$$
D W T(\mathrm{j}, \mathrm{k})=\int_{-\infty}^{\infty} x(t) \frac{1}{\sqrt{2^{j} \mid}} \psi\left(\frac{t-2^{j} k}{2^{j}}\right) d t
$$

where parameters $a$ and $b$ are replaced with $2^{j}$ and $2^{j} k$, respectively. This Continuous Wavelet Transformation has two disadvantages, namely redundancy and impracticability.

\section{B. Feature Extraction}

Feature extraction is a procedure used to produce new features that are used as independent variables in solving EEG signal classification problems. This procedure is carried out after translating the signals using the wavelet transformation method where the technique used in this study has been previously described. Some of the feature extractions used in this work is as follows [3], [15].

\section{1) Quartile}

This is a condition that divides a group of observations into four parts, with the value denoted by $\mathrm{Q}_{1}, \mathrm{Q}_{2}$, and $\mathrm{Q}_{3}$. This value has a variety of meanings where $\mathrm{Q}_{1}$ means that data falls under $25 \%, \mathrm{Q}_{2}$ means that it is below $50 \%$ while $\mathrm{Q}_{3}$ means that the data falls below $75 \%$ [16].

\section{2) Maximum value}

This is the highest value among the wavelet coefficients of each sub-band EEG signals.

\section{3) Minimum value}

This is the smallest value held between the wavelet coefficients of each sub-band EEG signals.

\section{4) Mean}

Mean is the average of each wavelet coefficient calculated from each sub-band.

\section{5) Standard deviation}

This is a value that describes the distribution of the value of the wavelet coefficient of each sub-band.

\section{Support Vector Machine}

Support Vector Machine (SVM) was first introduced by Vapnik in the 1960s as a classification method and has become a method often used in research because of its development in engineering and theory coupled with its existence in regression and estimation [17]. SVM has become an alternative method used to solve classification problems in machine learning and data mining. It is not like traditional statistical methods which empirically reduce errors but SVM aims to minimize error limits by maximizing the hyperplane margin used in solving classification problems [18]. SVM examines a hyperplane using support vector and margins described by the support vector. The hyperplane is a dividing line between classes, while margin is the shortest distance from a hyperplane with both sides of the class [19]. SVM is not only powerful but also known for binary classification tasks in machine learning for high dimensional feature vector due to its accuracy and capability to deal with a large number of predictors.

Suppose a case consists of two classes, given a data set D with $S=\left(\left(\mathbf{x}_{1}, y_{1}\right),\left(\mathbf{x}_{2}, y_{2}\right), \cdots,\left(\mathbf{x}_{i}, y_{i}\right)\right)$, where $\mathbf{x}_{i}$ has a connection with $y_{i}\left(\boldsymbol{x}_{i} \subseteq \mathbb{R}\right)$. Every $\mathrm{y}_{\mathrm{i}}$ is categorized +1 and -1 with $y_{i} \in\{+1,-1\}$. Figure illustrations are from two different classes separated by a hyperplane as shown below [19]:
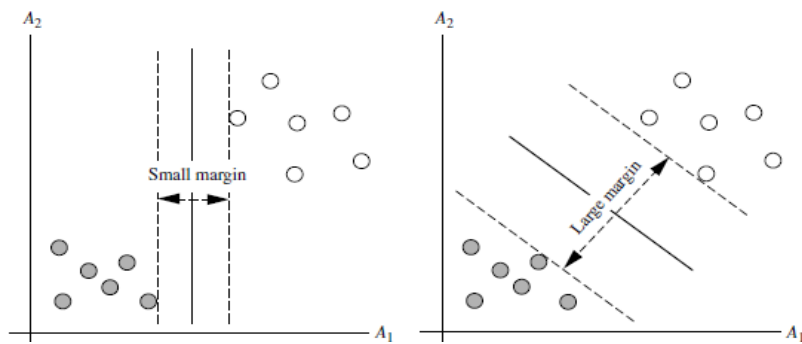

Fig. 1. Illustration of a hyperplane that might be on a classification.

The two forms of a hyperplane as shown in Fig. 1 produces different error values, however, there is no guarantee that they will show the same results when separating new data [20]. The hyperplane formula is written as follows:

$$
\mathbf{x}^{T} \mathbf{w}+b=0
$$

where $\mathbf{w}$ is a weight vector which is $\mathbf{w}=\left[w_{1}, w_{2}, \ldots, w_{3}\right], n$ is number variables and $\mathbf{x}^{T}$ is a matrix containing independent variables which is $\mathbf{x}^{T}=\left(x_{1}, x_{2}, \ldots, x_{n}\right)$. In addition, $\mathrm{b}$ is a scalar which has a bias.

In the case of non-linear, a different kernel is required to form a hyperplane. The kernel function is represented as $R^{n} \times R^{n}$ or regularly written as $K\left(x, x^{\prime}\right)=\left(\Phi(x), \Phi\left(x^{\prime}\right)\right)$. Some of the frequently used kernel's functions are as follows [21], [22]:

1) Kernel Linear

$$
K\left(\mathbf{x}, \mathbf{x}^{\prime}\right)=\mathbf{x}^{\prime} \mathbf{x}
$$

2) Kernel Polynomial

$$
K\left(\mathbf{x}, \mathbf{x}^{\prime}\right)=\left(\mathbf{x}^{\prime} \mathbf{x}+1\right)^{d}
$$

3) Kernel Radial Basis Function (RBF)

$$
K\left(\mathbf{x}, \mathbf{x}^{\prime}\right)=\exp \left(-\frac{\left\|\mathbf{x}-\mathbf{x}^{\prime}\right\|^{2}}{2 \sigma^{2}}\right)
$$




\section{Extreme Support Vector Machine}

Extreme support vector machine (ESVM) is a combination of methods of Support Vector Machine and Extreme Learning Machine. Extreme Learning Machine (ELM) is one of the available methods in Single Hidden Layer Feedforward Networks (SLFNs). The essence of ELM, which is the hidden layer on SLFNs, is not activated. Compared to other traditional computational techniques, it provides better results with a faster learning ability and least human intervention [23]. The performance of ELM is quite fast, but it does not look for the maximum hyperplane value. In otherwise, it minimizes the sum square error between classes, however it is not suitable for resolving classification cases. Therefore, it is necessary to combine SVM and ELM to obtain better results [24]. ESVM algorithm is as follows [24] [25]:

Input: Sample training: $\boldsymbol{x}_{i}=\left\{x_{i 1}, x_{i 2}, \ldots, x_{i p}\right\} \in \mathbf{R}^{p}, i=1, \ldots, n$. Label training: $y_{i} \in\{-1,+1\}$.

Output: Accuracy, Sensitivity, Specificity, AUC, Time, parameter.

1) Determine independent variables i.e. $\mathrm{A}=\left[x_{1}, x_{2}, \cdots, x_{n}\right]^{T}$ and dependent variable $y=\left[y_{1}, y_{2}, \cdots, y_{n}\right]^{T}$.

2) Divide data into training and testing.

3) Randomly produce a hidden layer parameter matrix $\mathbf{w}^{T} \in \mathbf{R}^{m \times(m+1)}$ and choose an activation function, typically the signum function, to calculate $\Phi\left(\mathbf{w}^{T}, \mathbf{A}\right)$ with formula as below:

$$
\Phi\left(\mathbf{w}^{T}, \mathbf{A}\right)=\left[\begin{array}{c}
\Phi\left(\mathbf{w}^{T}, \mathbf{x}_{1}\right)^{T} \\
\vdots \\
\Phi\left(\mathbf{w}^{T}, \mathbf{x}_{m}\right)^{T}
\end{array}\right]=\left\lfloor\begin{array}{c}
g\left(\mathbf{w}_{1}{ }^{T}, \mathbf{x}_{1}\right) \ldots g\left(\mathbf{w}_{m}{ }^{T}, \mathbf{x}_{1}\right) \\
g\left(\mathbf{w}_{1}{ }^{T}, \mathbf{x}_{n}\right) \ldots g\left(\mathbf{w}_{m}{ }^{T}, \mathbf{x}_{n}\right)
\end{array}\right\rfloor
$$

4) Generate $E_{\Phi}$ by computing $\left\lfloor\Phi\left(\mathbf{w}^{T}, \mathbf{A}\right),-\mathbf{e}\right\rfloor$ with $e$ is an $m \times 1$ vector of ones.

5) Calculate $\left\lfloor\begin{array}{l}\mathbf{w} \\ b\end{array}\right\rfloor$ by formula as follows:

$$
\left[\begin{array}{l}
\mathbf{w} \\
b
\end{array}\right]=\left(\frac{1}{c}+\left(\mathbf{E}_{\Phi}^{T} \mathbf{E}_{\Phi}\right)\right)^{T} \mathbf{E}_{\Phi}^{T} D e
$$

6) Classify new point using formula as defined follows:

$$
\Phi(x)^{T}-b\left\{\begin{array}{c}
>0, \text { then } x \in A+ \\
<0, \text { then } x \in A- \\
=0, x \in A+\text { or } x \in A-
\end{array}\right.
$$

\section{Methodology Research}

\section{A. Data Description}

The data applied in this research was recorded by EEG signals in epilepsy patients. It was obtained from Rumah Sakit Universitas Airlangga (RSUA) which had been labeled based on epilepsy sources in the frontal and temporal lobes. Throughout the EEG signal recording process, the object of research was stimulated for instruction by opening/closing eyes, hyperventilation and strobe light flashes. Data extracted from the results of EEG signal contained ictal and muscle artifact according to the notes given by medical personnel. EEG signal recording is carried out for 30 minutes and selected in such as way that it becomes a single signal data from an electrode with a duration of two seconds and with a sampling rate per of 500 per second, thereby, making the value 1000 points. The signal has filtered with a high pass value of $70 \mathrm{~Hz}$.

\section{B. Proposed Methodologies}

EEG signal detection through several processes. The EEG signals which originally had a duration of 30 minutes, it will be cutting for two seconds according to the signal characteristics such as ictal or muscle artefact. The next process can be seen in Fig. 2. Fig. 2 presents the block diagram of the proposed method.

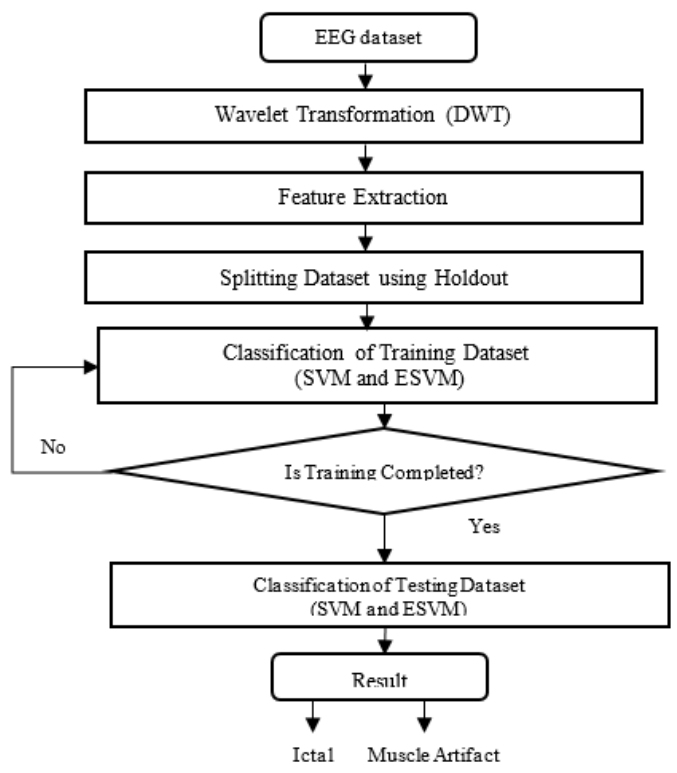

Fig. 2. Block diagram of the proposed epileptic EEG classification method.

\section{RESUlts}

In this section, the performances of ESVM are compared with various SVM kernel such as Linear, Polynomial, and Radial Basis Function (RBF) on EEG signal datasets. All experiments on ESVM, SVM with kernel Linear, SVM with kernel Polynomial, and SVM with kernel RBF for detection EEG signals are carried out in MATLAB environment running in Intel $\mathrm{CORE}^{\mathrm{TM}} \mathrm{i} 5$ and 4 GB DDR3 memory. For classification of EEG, 2160 signals are used, with the dataset consisting of 992 ictal and 1168 muscle artifact respectively.

In this study, the decomposition technique used is the Discrete Wavelet Transform (DWT) method, while the mother wavelet used is Daubechies, especially db2. The EEG signals are decomposed with DWT at 7 levels. Furthermore, the next process is the signal extraction process where the decomposed signals are extracted into several features. This is followed by classifying the EEG signals. The process of distributing classification data is randomly carried out and uses a holdout approach of 10 repetitions.

Fig. 3 shows illustration results of signal recording using EEG. The blue signal shows the structure of the ictal while the red signal plot shows muscle artifact. Both are visually 
difficult to distinguish because tools are required to reduce errors due to interpretation. Furthermore, in Fig. 3 shows the results of signal decomposition applying DWT.

(a)

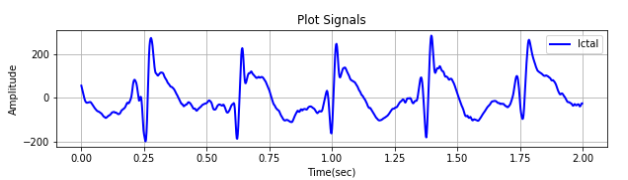

(b)

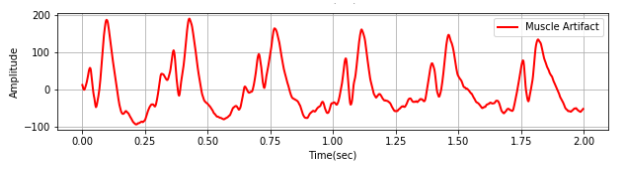

Fig. 3. Illustration of EEG signals (a) ictal (b) muscle artifact.

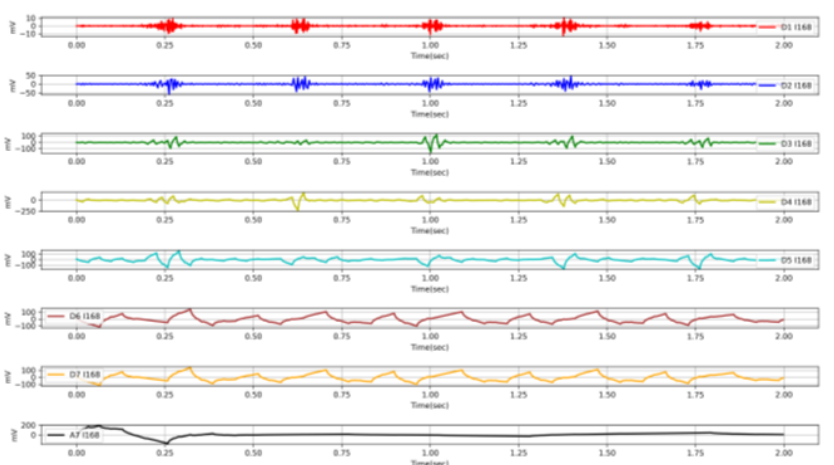

Fig. 4. Illustration of EEG signals after decomposition using DWT.

The decomposition process is used to determine the additional highlight of the characteristics of the EEG signal, with the decomposition process carried out in seven levels. This process decomposes the signal into low and high pass filter sub-signals. The high signal was eliminated, and the low decomposed into two processes. The level 1 decomposition process produces sub-signals A1 and D1, while level two produces sub-band A1, D1, A2, D2 etc. The results of the EEG signal decomposition were shown in Fig. 4 above.

In addition, the decomposition process conducted the next extraction steps. It previously explained that the features used in this study include quartiles 1,2 , and 3 , as well as maximum, minimum, mean and standard deviations. After decomposing and extracting constituents of level 7, 56 features were obtained. These were used as independent variables for the classification process.

TABLE I: MEAN OF ACCURACY, AREAN UNDER THE CURVE AND TIME CLASSIFICATION FOR TRAINING OF THE CLASSIFIER FOR VARIOUS HOLDOUT

\begin{tabular}{ccccc}
\hline Kernel & Holdout & Mean Accuracy & Mean AUC & Mean Time \\
\hline \multirow{4}{*}{ Linier } & $50: 50: 00$ & 0.94 & 0.94 & 0.01563 \\
\cline { 2 - 5 } & $60: 40: 00$ & 0.94 & 0.94 & 0.02344 \\
\cline { 2 - 5 } & $70: 30: 00$ & $\mathbf{0 . 9 5}$ & $\mathbf{0 . 9 5}$ & 0.00938 \\
\cline { 2 - 5 } Polynomial & $80: 20: 00$ & 0.94 & 0.94 & 0.00625 \\
\cline { 2 - 5 } & $50: 50: 00$ & 0.94 & 0.94 & 0.01878 \\
\cline { 2 - 5 } & $60: 40: 00$ & 0.94 & 0.94 & 0.02034 \\
\cline { 2 - 5 } & $70: 30: 00$ & 0.94 & 0.94 & 0.01565 \\
\hline \multirow{5}{*}{ RBF } & $80: 20: 00$ & 0.94 & 0.94 & 0.00939 \\
\cline { 2 - 5 } & $50: 50: 00$ & 0.92 & 0.92 & 0.05625 \\
\cline { 2 - 5 } & $60: 40: 00$ & 0.92 & 0.92 & 0.03752 \\
\cline { 2 - 5 } & $70: 30: 00$ & 0.93 & 0.93 & 0.0297 \\
\hline \multirow{5}{*}{ ELM } & $80: 20: 00$ & 0.92 & 0.92 & 0.02031 \\
\cline { 2 - 5 } & $50: 50: 00$ & 0.94 & 0.94 & $\mathbf{0 . 0 0 6 2 5}$ \\
\cline { 2 - 5 } & $60: 40: 00$ & 0.91 & 0.91 & $\mathbf{0}$ \\
\cline { 2 - 5 } & $70: 30: 00$ & 0.91 & 0.91 & $\mathbf{0}$ \\
\hline
\end{tabular}

Table I shows the performance of the SVM method using several types of kernels. In SVM method with linear kernel $C$ $=0.1$, SVM with polynomial kernel $C=0.1$ and $d=1$, SVM with $\mathrm{RBF}$ kernel $C=0.1$ and sigma $=300$, while SVM with ELM kernel $(E S V M) C=0.1$, hidden neuron $=1000$.

Data in Table I were divided into training and testing using the holdout validation wherein Table I shows that the performance given by each kernel varies in each percentage in accordance to the amount. The ratio of the distribution of training and testing is seen in Table I. This table also describes the generalization ability of the proposed method and the statistical relevance of data using holdout validation. It is observed that the classification accuracy has monotonically decreased when more samples was used in training than in testing the dataset. At each given ratio, SVM with Linear kernel produces Accuracy and AUC higher than other methods, especially the proportion of data 70:30. While for other data proportions, SVM with a polynomial kernel produces an average accuracy and AUC in proportion of 0.94 . It also describes that the proposed method is faster than the classifiers.

TABLE II: FERMORMANCE OF CLASSIFICATION VARIOUS SVM KERNELS

\begin{tabular}{cccccc}
\hline SVM & \multicolumn{5}{c}{ Training } \\
\cline { 2 - 6 } Kernel & Accuracy & Sensitivity & Specificity & AUC & Time (s) \\
\hline Linear & 0.952 & 0.964 & 0.941 & 0.953 & 42.57501 \\
\hline Poly & 0.951 & 0.963 & 0.941 & 0.952 & 38.00001 \\
\hline RBF & 0.946 & 0.975 & 0.922 & 0.948 & $\mathbf{0 . 2 8 5 9 4}$ \\
\hline ELM & $\mathbf{0 . 9 6 7}$ & $\mathbf{0 . 9 8 9}$ & $\mathbf{0 . 9 4 8}$ & $\mathbf{0 . 9 6 9}$ & 0.70784 \\
\hline SVM & \multicolumn{5}{c}{ Testing } \\
Kernel & Accuracy & Sensitivity & Specificity & AUC & Time (s) \\
\hline Linear & 0.943 & 0.967 & 0.924 & 0.945 & 0.01251 \\
\hline Poly & 0.942 & 0.957 & $\mathbf{0 . 9 3 0}$ & 0.943 & 0.00782 \\
\hline RBF & $\mathbf{0 . 9 4 7}$ & $\mathbf{0 . 9 7 2}$ & 0.927 & $\mathbf{0 . 9 5 0}$ & 0.0172 \\
\hline ELM & 0.921 & 0.934 & 0.908 & 0.921 & $\mathbf{0 . 0 0 3 1 3}$ \\
\hline
\end{tabular}

The classification and misclassification result of various SVMs used in categorizing EEG signals are given in Table II. The performance of the proposed approach has also been compared with various SVM techniques. The classification performance provided by the ESVM method with training data is better than other kernels. Accuracy, sensitivity, specificity and AUC values are 0.967, 0.989, 0.948 and 0.969 respectively. However, the performance provided by ESVM during testing is not as good as others. This is because the ESVM gives a faster execution time compared to others which have only 0.00313 . Based on the AUC value, the classifier method in this study is very nice for modelling EEG signal because its value is greater than $90 \%$.

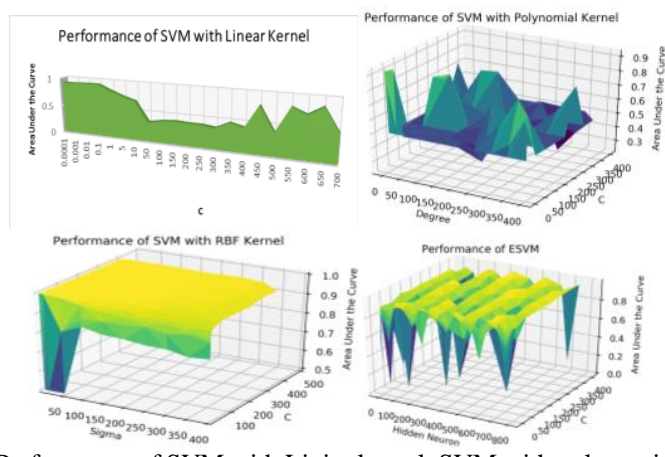

Fig. 3. Performance of SVM with Linier kernel, SVM with polynomial kernel, SVM with RBF kernel and ESVM. 
The figure above shows a plot of the performance of various kernels. The performance of SVM method with various types of kernels in Fig. 5 is different on each parameter applied. The plot shows different performance EEG signal detection patterns in each parameter.

\section{CONCLUSIONS}

The classifier approach has successfully classified complete of EEG dataset with emphasis on ictal and muscle artifact detection. ESVM achieves $92.1 \%$ classification accuracy and AUC and the executions time for classifier faster than other classifiers, which is absolutely convinced that this method is successful. Experiments show that the executions time of SVM classifier with ELM kernel is faster than SVM with Linier kernel and Polynomial kernel.

\section{CONFLICT OF INTEREST}

The author declare no conflict of interest.

\section{AUTHOR CONTRIBUTIONS}

Baiq Siska Febriani Astuti has written paper and analyze the data. Santi Wulan Purnami and R Mohamad Atok offered a useful suggestion for the paper preparation and writing this paper. Wardah Rahmatul Islamiyah has prepared data. Anda Iviana Juniani and Indah P. Wulandari have a useful suggestion for a target of the paper.

\section{ACKNOWLEDGMENT}

This work was supported by Program Direktorat Riset dan Pengabdian Masyarakat Institut Teknologi Sepuluh Nopember (No:5/E1/KP.PTNBH/20019 and 923/PKS/ITS/019).

\section{REFERENCES}

[1] M. A. Murugavel and S. Ramakrishnan, "Hierarchical multi-class SVM with ELM kernel for epileptic EEG signal classification," Medical \& Biological Engineering \& Computing, pp. 149-161, 2016.

[2] M. Diykh, Y. Li, and P. Wen, "Classify epileptic EEG signals using weighted complex networks based community structure detection," Expert Systems With Applications, pp. 87-100, 2017.

[3] R. E. Walpole, Pengantar Statistika, Jakarta: Gramedia Pustaka Utama, 1992.

[4] C. S. Burrus, R. A. Gopinath, and H. Guo, Introduction to Wavelets and Wavelet Transform, New Jersey: Prentice Hall, 1998.

[5] O. Faust, U. R. Acharya, H. Adeli, and A. Adeli, "Wavelet-based EEG processing for computer aided seizure detection and epilepsy diagnosis," Seizure, vol. 26, pp. 56-64, 2015.

[6] M. Li, W. Chen, and T. Zang, "Classification of epilepsy EEG signals using DWT-based envelope analysis and neural network ensemble," Biomedical Signal Processing and Control, pp. 357-365, 2017.

[7] I. Daubechies, Ten Lectures on Wavelets, Pennsylvania: The Society for Industrial and Applied Mathematics, 1992.

[8] F. A. Shah and L. Debnath, Wavelet Transform and Their Application, New York: Springer, 2015.

[9] S. W. Purnami, A. Embong, J. M. Zain, and S. P. Rahayu, "A new smooth support vector machine and its applications in diabetes disease diagnosis," Journal of Computer Science, vol. 5, pp. 1003-1008, 2009.

[10] J. Han And M. Kamber dan J. Pei, Data Mining: Concepts and Technique, 3rd ed., USA: Morgan Kaufman, 2012.

[11] Y. Kumar, M. L. Dewal, and R. S. Anand, "Epileptic seizure detection using DWT based fuzzy approximate entropy and support vector machine," Neurocomputing, vol. 133, pp. 271-279, 2014.

[12] P.-N. Tan, M. Steinbach, and V. Kumar, Introduction to Data Mining, Boston: Person, 2005.
[13] G.-B. Huang, D. H. Wang, and Y. Lan, "Extreme learning machines: A survey," Int. J. Mach. Learn. \& Cyber, pp. 107-122, 2011.

[14] B. Frénay and M. Verleysen, "Using SVM with randomised feature spaces: An extreme learning machine," in Proc. European Symposium on Artificial Neural Networks - Computational Intelligence, Bruges, 2010.

[15] Q. Liu, Q. He, and Z. Shi, "Extreme support vector machine classifier," Advances in Knowledge Discovery and Data Mining, pp. 222-233, 2008.

[16] W. Zhu, J. Miao, and L. Qing, Extreme Support Vector Regression, vol. 16, Heidelberg: Springer, 2014, pp. 25-34.

[17] A. T. Berg, "Definitions and classifications of epilepsies," Atlas of Epilepsies, London, Springer, 2010, pp. 1-22.

[18] A. Tandle and N. Jog, "Classification of artefacts in EEG signal recordings and overview of removing techniques," International Journal of Computer Applications, pp. 0975-8887, 2015.

[19] X. Liu, L. Wang, G.-B. Huang, J. Zhang, and J. Yin, "Multiple kernel extreme learning machine," Neurocomputing, vol. 149, pp. 253-264, 2015.

[20] C. Cortes and V. Vapnik, "Support vector networks," Machine Learning, pp. 273-297, 1995 .

[21] M. Li, W. Chen, and T. Zang, "Automatic epilepsy detection using wavelet-based nonlinear analysis and optimized SVM," Biocybernetics and Biomedical Engineering, pp. 708-718, 2016.

[22] M. Li, W. Chen, and T. Zhang, "Automatic epileptic EEG detection using DT-CWT-based non-linear features," Biomedical Signal Processing and Control, vol. 34, pp. 114-125, 2017.

[23] O. Kocadagli and R. Langari, "Classification of EEG signals for epileptic seizures using hybrid artifi-cial neural networks based wavelet transforms and fuzzy relations," Expert Systems With Applications, pp. 419-434, 2017.

[24] N. Deng, Y. Tian, and C. Zhang, Support Vector Machines: Optimization Based Theory, Algorithms, and Extensions, Boca Raton: Taylor \& Francis Group, 2013.

[25] B. Scholkopf and A. J. Smola, Learning with Kernels: Support Vector Machines, Regularization, Optimization, and Beyond, London: The MIT Press, 2002.

[26] D. P. Wulandari, Y. K. Suprapto, F. T. Elyantono, W. S. Purnami, and W. R. Islamiyah, "Visualization of epilepsy patient's brain condition based on spectral analysis of EEG signals using topographic mapping," in Proc. 2018 International Conference on Computer Engineering, Network and Intelligent Multimedia (CENIM), Surabaya, 2018.

Copyright ( $(2020$ by the authors. This is an open access article distributed under the Creative Commons Attribution License which permits unrestricted use, distribution, and reproduction in any medium, provided the original work is properly cited (CC BY 4.0).

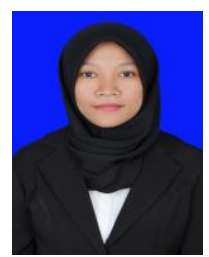

Baiq Siska Febriani Astuti was born in Lombok on February 22, 1994. She recived her bachelor degree from the Departement of Statistics, Islamic University of Indonesia. Indonesia in 2016. She is currently a graduate student at Institut Teknologi Sepuluh Nopember. Her research interests include neural network and machine learning.

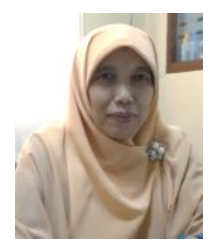

Santi Wulan Purnami was born in Kediri on September 23, 1972. She recived her bachelor degree from the Departement of Statistics, Institut Teknologi Sepuluh Nopember (ITS). Indonesia in 1996. She got the M.Si degree in Gajah Mada University, Indonesia in 2004. She recived the $\mathrm{Ph} . \mathrm{D}$ degree in computer science from University Malaysia Pahang. Malaysia in 2011.

She is a senior lecturer at the Department of Statistics, Faculty of Mathematics, Computing and Data Science, ITS from 1998 until now. Her research interests include in biostatistics, machine learning and support vector machine. She has been granted several research funds in the above-mentioned areas.

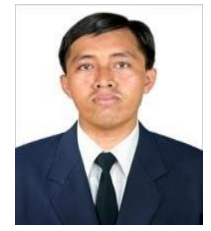

R Mohamad Atok was born in Malang, Indonesia on September 15, 1971. Atok graduated his PhD in 2016 from UKM, Malaysia on applied statistics.

$\mathrm{He}$ is a lecturer at the Department of Statistics, Institut Teknologi Sepuluh Nopember (ITS) from 1996 until now. Since 2017 he has served as the head of Statistics Economics, Financial and Actuarial Science Laboratory. 


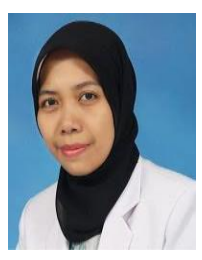

Wardah Rahmatul Islamiyah was born in Surabaya on May 19, 1979. She recived the bachelor degree from the Faculty of Medicine, Brawijaya University, Indonesia in 2004. She continued her study to pursue specialist from Airlangga University. Indonesia in 2009

She has been an academic staff at the Department of Neurology, Airlangga University, since 2009 and she has been a doctor in Rumah Sakit Universitas Airlangga. Her research interest in epilepsy. She has been involved in several research groups related epileptic seizure.

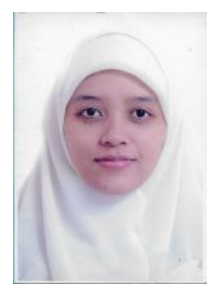

Diah P. Wulandari was born in Surabaya on December 19, 1980. She received her bachelor degree from the Department of Electrical Engineering, Institut Teknologi Bandung, Indonesia, in 2004. She got the M.Sc degree from the School of Informatics, University of Edinburgh, United Kingdom, in 2006. Her doctoral degree was awarded from the Department of Electrical Engineering, Institut Teknologi Sepuluh Nopember (ITS) in 2016. She focused her dissertation on audio signal processing.

She has been an academic staff at the Department of Computer Engineering, ITS, since 2005. Her research interest is in the field of audio and biomedical signal processing, with the implementation of machine learning techniques to overcome problems in these areas. She has been involved in several research groups related to machine listening, and computational neuroscience, particularly in the case of epilepsy disease.

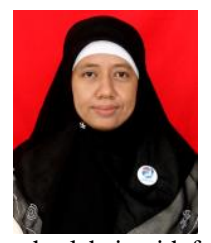

Anda Iviana Juniani graduated from Industrial Engineering, Institut Teknologi Sepuluh Nopember in 2010. She has been a lecturer in the Department of Design and Manufacture since 2004 and a member of PEI (Perhimpunan Ergonomi Indonesia). Her research interest is in risk assessment, ergonomic product design and reliability analysis. Currently she is working on design wheelchair with foot cycling function for stroke patients rehabilitation. She has been also involved in several research groups related to epilepsy disease treatment of experimental design. 